\title{
IEGGR: Improved Energy-aware and Delivery Guarantee Geographic Routing
}

\author{
Kenza Redjimi, Mehdi Boulaiche, and Mohammed Redjimi
}

\begin{abstract}
Wireless Sensor Networks are a set of sensor nodes that probe their surroundings and send their data over multi-hop routes to a base-station (BS) for processing. Many routing protocols have been developed for WSNs. A great attention has been given to geographic routing protocols, which rely only on local knowledge of nodes to disseminate data towards the BS. In this paper, we propose a new energy efficient geographic routing protocol called Improved Energy-aware and delivery Guarantee Geographic Routing protocol (IEGGR). The proposed protocol exploits the positions of neighboring nodes, which are closest to the BS to forward packets through the shortest path in terms of energy consumption. IEGGR introduces a mechanism in order to forward packets efficiently around voids. IEGGR algorithm considers the WSN as a connected-graph and to route a packet from a source node to the BS, IEGGR tries making the locally optimal choice at each intermediate node with the hope of finding a global optimum path, which aims to minimize energy consumption. To find greedily the optimal path, each forwarding node exploits its neighbor positions that are closest to the BS in order to iteratively building the end-to-end optimal path. Simulation results show that IEGGR is efficient in energy management during packet forwarding.
\end{abstract}

Index Terms-Energy consumption, Energy efficiency, Geographic routing protocol, GPS localization, Wireless sensor network.

\section{INTRODUCTION}

$\mathrm{W}$ ireless Sensor Networks (WSNs) [1-3] are a set of sensor nodes that probe their surroundings and send their data over multi-hop routes to a base-station (BS) for processing. Thus, in these networks, each node is capable of detecting its environment and processing information locally or sends it to one or more collecting point. These sensors can monitor a variety of conditions: temperature, humidity, pressure, the presence or absence of certain types of objects, etc. Consequently, WSNs find applications in a wide range of domains such as health applications [4-5], smart homes,

Manuscript received December 14, 2020; revised March 4, 2021. Date of publication May 31, 2021. Date of current version May 31, 2021. The associate editor prof. Korhan Cengiz has been coordinating the review of this manuscript and approved it for publication.

Authors are with the University 20 Aout 1955, Department of Computer Science, 21000 Skikda, Algeria (e-mails: \{k.redjimi, m.boulaiche, m.redjimi\}@univ-skikda.dz).

Digital Object Identifier (DOI): 10.24138/jcomss-2020-0019 smart cities [6], smart parking [7-8], industrial automation [9], traffic monitoring [10-11], military field [12-13], security surveillance [14], agriculture monitoring [15] and many other application's fields [16]. Several works and several solutions were proposed to improve the functioning of these networks. Nevertheless, several problems still need to be addressed again and several solutions need to be improved. The energy management is among the major problems in wireless sensor networks. The concern is the energy consumed by each node. Energy consumption in WSNs is a very important metric that influences directly the network lifetime. A sensor node expends its maximum energy in data communication. In order to conserve node energy and so increase the network lifetime, energy consumption should be taken into consideration in all levels of the network architecture (physical, MAC, and Network) [17]. Thus, protocol developers should integrate this constraint when developing a new communication protocol. Many routing protocols have been developed for WSNs. Most of these routing protocols require nodes localization information since a sensed event is directly related to the node's location. This information is required to calculate distance between two particular nodes such that energy consumption can be estimated. Because there is no addressing system for wireless sensor networks (like IP addressing) and because they are deployed randomly in a region, the location information of these nodes can be used in routing data efficiently in terms of energy. For example, if the region to sense is known by using nodes localization in order to broadcast the request to this particular region, it is easy to eliminate the number of retransmissions.

In geographic routing [18], a node selects a neighbor, which is closer to the destination as a next hop. Although it is simple and efficient, this routing technique fails when there is no neighbor closer to the destination than the current node. This issue is called the void problem (also known as local minima problem).

Our work is particularly interested to design a new geographic routing protocol that addresses the two major problems of geographic routing: energy consumption and delivery guarantee towards the base station. In this way, we will present a new geographic routing protocol that forwards packets through the optimal path in terms of energy 
consumption between the source node and the base station based only on local knowledge of nodes (neighborhood). Our protocol increases packets delivery ration to the base station.

The rest of this article is organized as follows: section II presents related work on geographic routing protocols in WSNs. In section III, we present the network model. Our protocol IEGGR is presented in section IV, and simulation results of our protocol are discussed in section V. Section VI concludes this article.

\section{RELATED WORK}

Routing in wireless sensor networks is a cooperative task in which all nodes are expected to participate in order to forward packets toward the BS. Therefore, protocols designed for data routing in such networks are different from those designed for routing in other types of networks. Routing protocols designed for wireless sensor networks have to deal with the characteristics of such networks. These protocols have to be designed to minimize communication overhead since sensor nodes have limited resources. They also need to deal with the number of nodes within the network, and very importantly, a routing protocol designed for such networks should minimize energy consumption since nodes are equipped by batteries, which are not replaceable.

Routing protocols for wireless sensor networks can be categorized into three types, namely: flat routing, hierarchical routing, and geographic routing. Flat routing protocols include reactive protocols such as Flat routing protocols can be reactive (On-Demand) protocols such as Dynamic Source Routing (DSR) [2], and Ad hoc On-demand Distance Vector (AODV) [19-21] or proactive (Table-Driven) protocols such as Destination Sequenced Distance Vector (DSDV) [22]. In hierarchical routing, nodes are divided into clusters and a cluster head is assigned to each cluster head [23-24]. Lowenergy adaptive clustering hierarchy (LEACH) [25-26] and its variants are typical examples of hierarchical routing protocols. In geographic routing protocols, the position information of nodes is used to forward packets toward the final destination. Greedy Perimeter Stateless Routing GPSR is an example of geographic routing protocols GPSR [27].

Geographic routing has become an attractive solution [28-34] for wireless sensor networks where nodes keep only information about local one-hop neighbors. In geographic routing, a node selects a next forwarding node based only on the location of itself, its neighbors and the base station. The location information can be obtained with GPS or through any other localization system. As it does not use control packets to establish a path, the geographic routing reduces routing control overhead flooded in the network to maintain network connectivity compared with other types of routing protocols. So, the areas which make the best use of communications between neighboring nodes are those where geographic protocols can be successfully applied; this is the case with the Internet of Things (Iot) [35-37] and the Vehicular Ad hoc Networks (VANETs) [38] among others.

Protocols called greedy [39] forward packets such that their routes be the closest to the path as the crow flies between the source and the destination. Nearest with Forward
Progress (NFP) [40-42] protocol selects its closest neighbor among those in the direction of the destination to forward the packet. Whereas, with Most Forward within the transmission Range (MFR) protocol [39],[43] a forwarding node selects its neighbor that is closest to the destination as next forwarding node. The Normalized ADVance (NADV) protocol [44] selects the neighbor with the optimal trade-off between the advance and link cost.

Although simple and generate little overhead in auxiliary routing structure maintenance, greedy routing algorithms fail when there is no neighbor closer to the destination than the current node. This issue is called the void problem (also known as local minima problem). Void problem is a frequent problem in underwater sensor networks and in networks with low density [45-49]. Routing based on planar graph is a geographic routing strategy, which is able to overcome greedy routing problem (voids problem). A graph is said to be planar if it can be drawn in the plane so that its edges intersect only at their ends [50]. A planar graph forms a set of faces as illustrated in Figure 1 and routing is performed by forwarding packets along the interiors of a sequence of adjacent faces, which provide progress toward the destination, applying the right (or left) hand rule [51-52]. Authors in [51] and [27] proposed to combine a greedy algorithm and face routing (figure 1). The principle is simple: initially, the packet is forwarded using greedy mode but if the packet reaches a void, this packet is balanced to face routing mode to forward it around the void. Then, the packet is returned to greedy mode as soon as possible. J. Kuruvila et al. [53] proposed an extension to GFG method, w h i c h models energy consumption. Before forwarding a packet to a node $v$, node $u$ checks if there is a node $w$ neighbor to both nodes $u$ and $v$, such that energy consumption is less if the packet follows the path $u w v$ rather than the direct path $u v$. If it is, $u$ sends the packet to $w$, which will process in the same way.

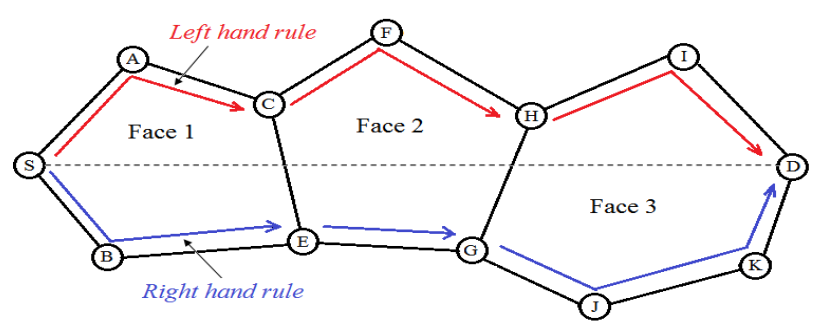

Fig. 1. Face routing.

In geographic routing protocols, nodes select as next hop relay the most promising neighbor (according to some metrics) among those being closer to the destination than themselves. If nodes know the positions of all other nodes in the whole network, so the optimal path that minimizes end-to-end energy consumption can be calculated using Dijkstra's shortest path algorithm [54], where transmission cost between two nodes represents the cost of the edge between these nodes. However, nodes know only their positions, the positions of their neighbors, and the position of the base station. In this work, we will propose a new 
localized and energy efficient geographic routing protocol. The proposed protocol improves EGGR [31] protocol in the following points:

1) Rather than assuming that the retransmission between each neighbor and the BS is optimal, IEGGR tries making the locally optimal choice with the hope of finding a global optimum path without any assumptions.

2) Rather than calculating the next hop for each packet, next hop is calculated each round in order to reduce energy consumption and calculation overhead.

3) No information about neighbor status is updated and another strategy is proposed in order to forward packets around a void through the optimal path as well.

4) The local sub-graph $G^{\prime}$ is constructed only at network setup since sensor nodes are static in order to reduce energy consumption and calculation overhead. However, edge weights are recalculated at each round in order to balance the role of next forwarding neighbor among multiple neighbors.

\section{NETWORK MODEL}

We consider a wireless sensor network in which all nodes are static and energy-limited. It is assumed that each node is aware of its own position, neighbor positions, the position of the base station, and the remaining energy of its neighbors. The location information can be obtained by equipping nodes with GPS or through any other localization system. Neighbor's location information and remaining energy information can be obtained by periodic beacon messages. We also assume that sensor nodes communicate through the wireless medium and all radios are bidirectional, two sensor nodes can communicate directly with each other if they are within their transmission ranges. Each node has a maximum transmission range $r$ that can be considered, without losing generality, the same for all nodes. Sensor nodes perform sensing, transmission, and relaying packets to the base station. We represent a wireless sensor network as a connected unit disk graph $G=(E, V)$, where the edge set $E$ corresponds to the wireless links between nodes and the vertex set $V$ corresponds to nodes in the network. In our protocol, we will adopt the energy model of Ivan Stojmenovic et al. [55] where he generalized Rodoplu and Meng's model [56] by adding a linear factor ' $a$ ' and assumed that the power needed for the transmission and the reception of a signal is:

$$
u(d)=a d^{\alpha}+c
$$

where $d$ is the distance between the two nodes, $\alpha$ is the media attenuation factor (between 2 and 6), $c$ a constant (may also include the energy consumed in computer processing and encoding/decoding at each node), and $a$ can be adjusted to the physical environment, unit of length, unit size of a signal,...

\section{IMPROVED EGGR}

The main idea of IEGGR algorithm is to consider the WSN as a connected-graph. Then, to route a packet from a source node to the BS, IEGGR tries making the locally optimal choice at each intermediate node with the hope of finding a global optimum path between the source node and the base station. This optimal path aims to minimize energy consumption. To find greedily the optimal path, each forwarding node exploits its neighbor positions that are closest to the BS in order to iteratively building the end-to-end optimal path.

\section{A. The Approach Overview}

In geographic routing, a node selects a neighbor, which is closer to the base station as a next hop. Although it is simple and efficient, this routing technique does not allow forwarding packet through the optimal path in terms of energy consumption. In addition, this technique fails when there is no neighbor closer to the destination than the current node. This issue is called the void problem (also known as local minima problem).

The main idea of IEGGR is to initiate a Minimal Spanning Tree (MST) calculation process. Basically, each node constructs a local sub-graph $G^{\prime}=\left(E^{\prime}, V^{\prime}\right)$, including neighbors, which are in the direction of the BS, that is, neighbors which are closest to the BS than the source node (see subsection B). We refer to such area as the Routing Area. Then, a minimal spanning tree is calculated on the local sub-graph $G^{\prime}$ using Prim's algorithm [57]. This step gives a set of paths starting from the current node towards a set of leaves. The next hop neighbor is the first node on the path (towards one of these leaves) where the sum of its weights is the minimum (see subsection D).

A node that encounters a void towards the BS should widen its sub-graph area by $\delta$ degrees to include other neighbors. We refer to such area as the Recovery Area. Then, it applies the same procedure and uses source routing to forward packets, that is, the shortest path is included in packet's header and next hop forwards the packet according to the path included in packet's header. All these phases are summarized in algorithm1.

Obviously two issues arise, namely, (1) how the local subgraph $G^{\prime}$ is defined, and (2) how big a node should widen its recovery area, and in this case how the packet should be forwarded through this area to go around the void. These two issues are in essence inter-related and depend on how greedy and recovery mode is used to successfully forward a packet until the BS. Greedy routing technique opts to advance packets toward the BS at each hope. Thus, to construct the local sub-graph $G^{\prime}$, only links that allow advancing a packet towards the BS should be considered. On the other hand, recovery mode opts to forward a packet around a void and returns back to greedy mode as soon as possible. Thus, the size of the recovery area is subject to trade-off. From the recovery point-of-view, it is advantageous to make the recovery area quite large and wide in all directions. However, a very wide recovery area can go with the packet away from the BS and so increase both energy consumption and end-to-end transmission delay. Therefore, our approach constrains the width of the recovery area so that the energy consumption and end-toend transmission delay can be minimized.

We define the set $A_{1-h o p}$ to be the nodes that can reach the node $A$ directly. Assuming a free space radio propagation model, a node $X \in A_{l-h o p}$ if $X$ is at most $r$ units away from the 
node $A$, where $r$ is the sensor node's communication range. Thus, the routing area of a node $A$ as depicted Figure 2, is the set of nodes which are closest to the BS. In that case, all packets received from outside $A$ 's Routing Area will be routed through nodes that are inside $A$ 's Routing_Area. On the other hand, if the routing area is empty, i.e., no neighbor is closest to the BS than the current node; all packets received from outside A's Routing_Area will be routed through nodes that are inside A's Recovery_Area (see Figure 3).

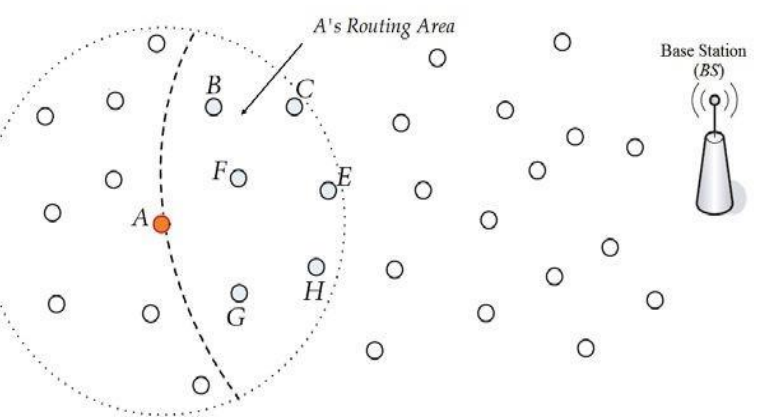

Fig. 2. Routing area.

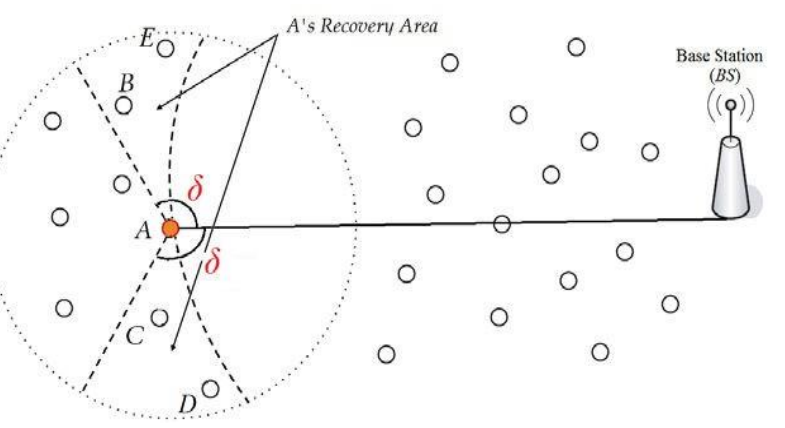

Fig. 3. Recovery area.

The above observation, which is illustrated in Figure 2, concludes that the local sub-graph $G$ ' constructed from nodes in the Routing Area differs from that constructed from nodes in the Recovery Area. In addition, routing technique applied inside Routing Area differs from that applied inside Recovery Area.

\section{B. Sub-graph Formation Algorithm}

In greedy routing techniques, each node is expected to participate in the process of forwarding packets towards the BS. Therefore, at network setup time, each node initiates the process of selecting its next forwarding neighbor. Fundamentally, this neighbor is used to forward all received packets. To balance the load and avoid overburdening a single neighbor, the next forwarding neighbor role is periodically reselected among multiple neighbors, where a neighbor serves as the next forwarding neighbor in a round; consequently, the process of selecting the next forwarding neighbor is reinitiated each round. The time duration of a round would naturally depends on the traffic intensity in the network. A node also can trigger the role rotation when its next forwarding neighbor energy supply runs low. Each node maintains a neighbors table containing: neighbor's identifier, neighbor's position and neighbor's remaining energy. Recall that each node periodically broadcasts a beacon message containing its position and remaining energy. The local subgraph is the graph $G^{\prime}=\left(E^{\prime}, V^{\prime}\right)$ such that: $V^{\prime}$ corresponds to the set of neighbors in $A_{\text {Routing_Area }} / A_{\text {Recovery_Area. An }}$ And $E^{\prime}$ corresponds to the set of edges between these neighbors. Let $\operatorname{dist}(u, v)$ be the distance between the two nodes $u$ and $v$ and $r$ is the sensor node's communication range.

\section{Algorithm 1: Routing and Recovery area algorithm}

$s:$ the node that is handling packet $P$;

$N(s)$ : the set of $s$ 's neighbors which are closest to the BS;

$R(s)$ : the set of $s$ 's neighbors which are in the recovery area;

$H(s)$ : the set of $s$ 's one hop neighbors;

For each node $u \in H s$ Do

$$
\begin{aligned}
& a=\operatorname{dist}(s, u) ; \\
& b=\operatorname{dist}(s, B S) ; \\
& c=\operatorname{dist}(u, B S) ; \\
& \text { If } \operatorname{dist}(u, B S) \leq \operatorname{dist}(s, B S) \text { Then } \\
& \quad \operatorname{Add} u \text { to } N(s) ;
\end{aligned}
$$

\section{EndIf}

$$
\text { If }\left[\left(b^{2}+c^{2}-a^{2}\right) /\left(2 * b^{*} c\right)\right] \leq \delta \text { Then }
$$

Add $u$ to $R(s)$;

\section{EndIf}

EndFor

1) Routing Area sub-graph

- $\quad$ A node $u \in V^{\prime}$ if and only if dist $(u, B S) \leq \operatorname{dist}(s$, $B S)$ and $\operatorname{dist}(s, u) \leq r$.

An edge $u v \in E^{\prime}$ if and only if $u, v \in V^{\prime}$ and $\operatorname{dist}(s$, $u) \leq r$ and $\operatorname{dist}(s, v) \leq r$ and $\operatorname{dist}(u, v) \leq r$ and $\operatorname{dist}(s$, $u) \leq \operatorname{dist}(s, v)$.

- Plus an edge between the current node $s$ and each node $u \in V^{\prime}$.

Where $s$ is the current node and $B S$ is the base station.

\section{2) Recovery Area sub-graph}

Let $a$ be the distance between $s$ and $u, b$ the distance between $s$ and the $B S$, and $c$ the distance between $u$ and the BS:

- A node $u \in V$, if and only if $\left[\left(b^{2}+c^{2}-a^{2}\right) /\left(2 * b^{*} c\right)\right] \leq \delta$ An edge $u v \in E^{\prime}$ if and only if $u, v \in V^{\prime}$ and $\operatorname{dist}(s$, $u) \leq r$ and $\operatorname{dist}(s, v) \leq r$ and $\operatorname{dist}(u, v) \leq r$ and $\operatorname{dist}(v$, $B S) \leq \operatorname{dist}(u, B S)$.

- Plus an edge between the current node $s$ and each node $u \in V^{\prime}$.

where $s$ is the current node, $B S$ is the base station, and $\delta$ is the width angle of $S_{\text {Recovery_Area. }}$

These two steps are summarized in Algorithm 1. 


\section{Edges Weights}

The weight of each edge represents the energy required for the transmission and reception between the two nodes. The cost of transmission/reception between two nodes $u, v \in V$, and the transmission/reception cost between the current node $s$ and each node $u \in V^{\prime}$ is calculated according to equation (1).

To balance energy consumption and to regularly distribute it on all neighbors, we propose to add another metric, which is the remaining energy $R E_{u}$ of neighbor nodes. So, the weight of each edge is based on two parameters; the transmission/reception energy and the remaining energy of neighboring nodes:

$$
\text { Edge } \_ \text {weight }=\frac{\left(a d^{\alpha}+c\right)}{R E_{u}}
$$

where $d$ is the distance between the two nodes, $\alpha$ a media attenuation factor between 2 and $6, c$ is a constant, and $a$ : can be adjusted to the physical environment, unit of length, unit size of a signal.

\section{Algorithm 2: Local sub-graph G'algorithm}

$s:$ the node that is handling packet $P$;

$N(s)$ : the set of $s$ 's neighbors which are closest to the BS; $R(s)$ : the set of $s$ 's neighbors which are in the recovery area; $H(s)$ : the set of $s$ 's one hop neighbors;

$$
\text { If } N(s) \neq \phi \text { Then }
$$

For each node $u \in N(s)$ Do

If $\operatorname{dist}(u, B S) \leq \operatorname{dist}(s, B S)$ Then

\section{EndIf}

Add Edge $s u$ to $E^{\prime}$;

\section{EndFor}

For each two nodes $u$ and $v \in N(s)$ Do

If $\operatorname{dist}(s, u) \leq \operatorname{dist}(s, v)$ Then

EndIf

Add Edge $u v$ to $E$,

\section{EndFor}

For each node $u \in R(s)$ Do

If $\operatorname{dist}(u, B S) \leq \operatorname{dist}(s, B S)$ Then

EndIf

Add Edge $s u$ to $E$ ';

\section{EndFor}

For each two nodes $u$ and $v \in R(s)$ Do

If $\operatorname{dist}(s, u) \leq \operatorname{dist}(s, v)$ Then

EndIf

Add Edge $u v$ to $E$,

\section{EndFor}

EndIf

It is worthy to recall here that the local sub-graph $G^{\prime}$ is constructed only at network setup since sensor nodes are static. However, edge weights are recalculated at each round in order to balance the role of next forwarding neighbor among multiple neighbors.

\section{Next Forwarding Neighbor Setup}

As stated earlier, after constructing the local sub-graph $G$ ' a node applies Prim's algorithm [21] in order to find the Minimum Spanning Tree. It is important to emphasize that this algorithm provides the optimal solution to the Minimum Spanning Tree problem. The structure of the Prim's algorithm for the local sub-graph $G^{\prime}$ is summarized in algorithm 2. A spanning tree is a sub-graph of an undirected graph that contains all the vertices and has no cycles. The sum of an MST's weights is the minimum possible. As illustrated in algorithm 3, the MST consists of a set of minimum paths towards the BS in terms of energy consumption. The next forwarding neighbor is the first node on the path with the minimum sum of weights as illustrated in Figure 6.

Algorithm 3: Prim's algorithm [57]:

1. Choose current node $S$ to be the root node. Set $V t=\{S\}$ and $E t=\varnothing$.

2. Find an edge with the smallest weight such that one of its end points is in $V t$ and the other is in $V \backslash V t$. Add this edge to $E t$ and its new vertex to $V t$.

3. If $V \backslash V t=\emptyset$, then terminate. Otherwise, go back to Step 2.

4. The minimum Spanning Tree is $M S T=(V t, E t)$.

\section{E. Illustrative Example and Analysis}

To illustrate the void formation process, let us consider the example topology in Figure 4. At network setup time, node A initiates the process of selecting its next forwarding neighbor towards the $\mathrm{BS}$. The routing area of node $\mathrm{A}$

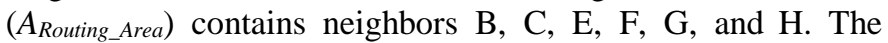
first step in selecting next forwarding neighbor is sub-graph formation. The sub-graph formed by node $\mathrm{A}$ is illustrated in Figure 4.b. Let us assume that the corresponding edge weights are as illustrated in the figure. After formation of sub-graph, node A applies Prim's algorithm to find the MST from the sub-graph. The MST calculated is illustrated in blue in figure 4.c. The set of paths towards the BS obtained is:

1. AFBC with a total weight of: $3+2+3=8$.

2. AFBE with a total weight of: $3+2+2=7$.

3. AGH with a total weight of: $4+2=6$.

The path having the minimum weight is AGH so the next forwarding neighbor of node $\mathrm{A}$ is $\mathrm{G}$. node $\mathrm{A}$ forward all received packets through node $G$ until the round duration expires.

On the other hand, if node $\mathrm{A}$ has no neighbor closer to the BS, it will use its recovery area to form the sub-graph. As illustrated in Figure 5. a, the recovery area of node $\mathrm{A}$ (A $A_{\text {Recovery_Area }}$ ) contains neighbors B, C, D. 


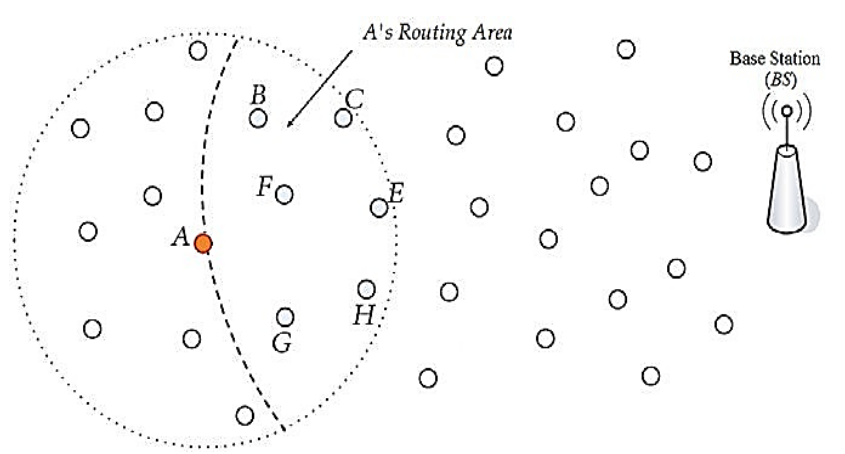

(a)

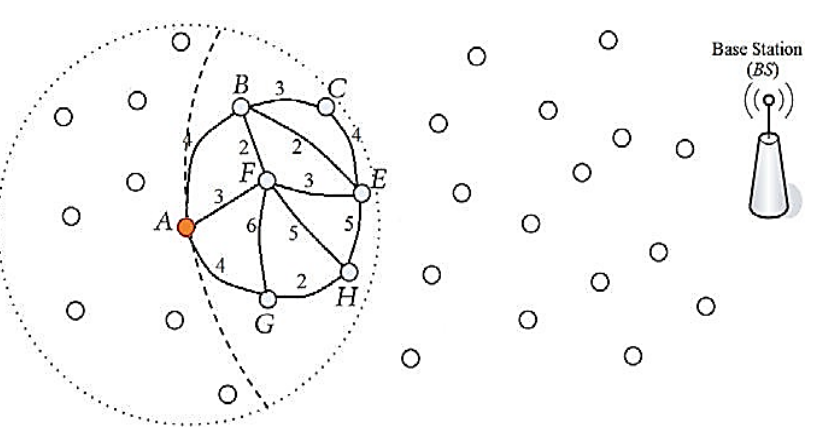

(b)

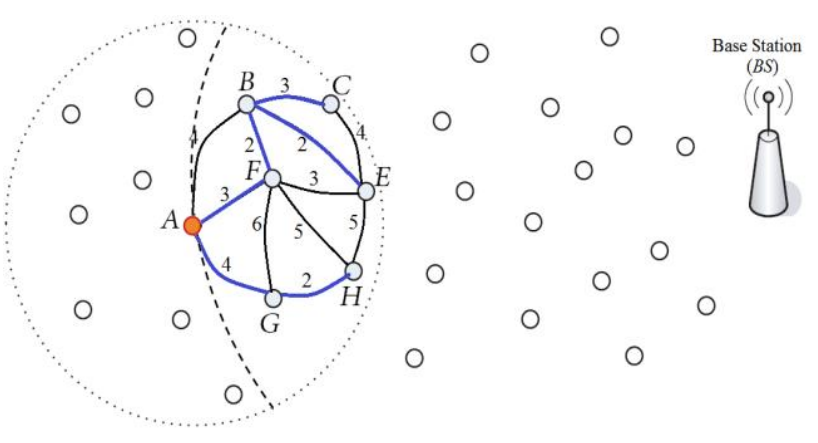

(c)

Fig. 4. Local sub-graph $G^{\prime}$.

In this case, the local sub-graph formed by node $\mathrm{A}$ is illustrated in Figure 5. b. Let us assume that the corresponding edge weights are as illustrated in the figure 5 .

After applying Prim's algorithm, the MST obtained is illustrated in blue in Figure 5.c.

The set of paths on the MST are:

1. ABE with a total weight of: $5+2=7$.

2. ACD with a total weight of: $2+2=4$.

The path having the minimum weight is ACD so the next forwarding neighbor of node $\mathrm{A}$ is $\mathrm{C}$. Node $\mathrm{A}$ forward all received packets in recovery mode through node $\mathrm{C}$ until the round duration expires.

\section{F. Routing Strategy}

In a wireless sensor network, data packets are to be forwarded from source nodes to the BS through multi-hop routes. A packet has two states in IEGGR, namely, normal mode and recovery mode. The normal mode allows

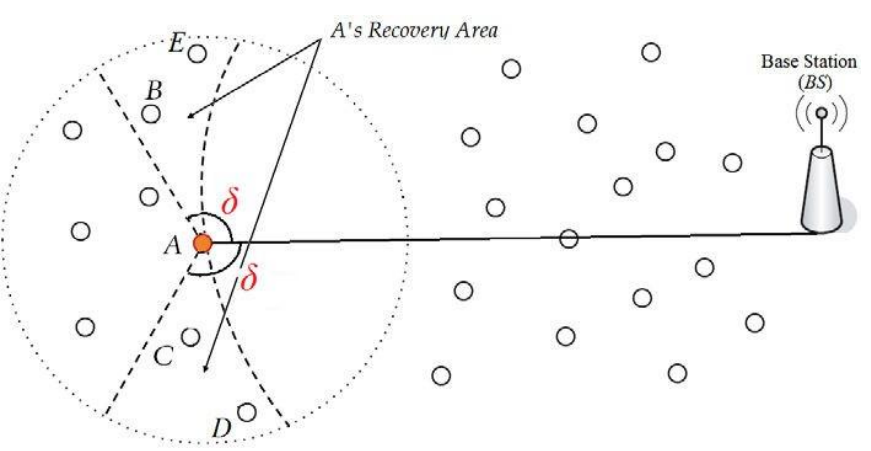

(a)

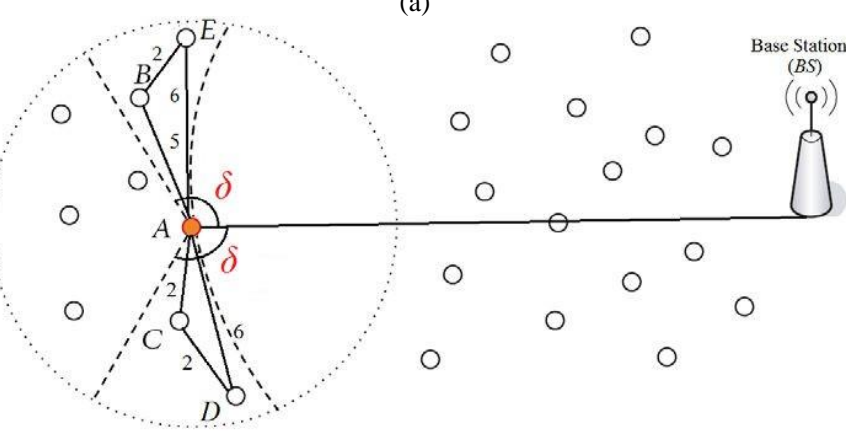

(b)

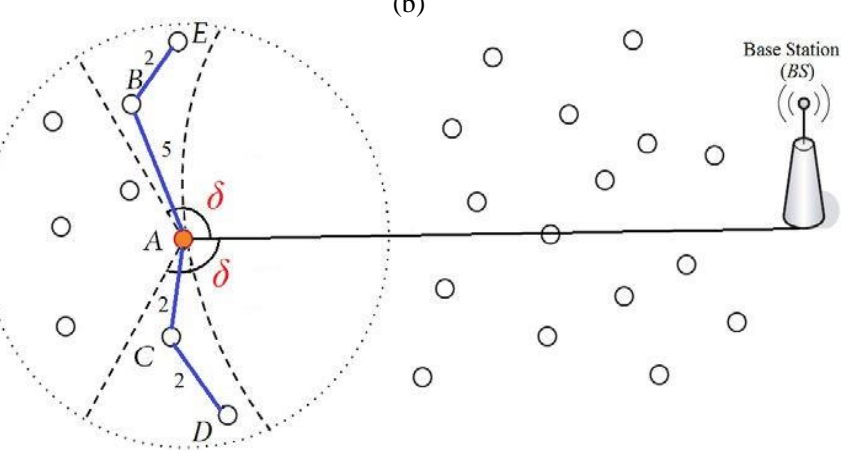

(c)

Fig. 5. Local sub-graph G' constructed from the recovery area.

forwarding packets through Routing Area whereas recovery mode allows forwarding packets through Recovery Area. Initially, a packet is forwarded in normal mode and whenever the packet encounters a void at a node $p$, it will be balanced to recovery mode to forward it around the void. When the packet gets to a node $q$ having at least a neighbor closer to the $\mathrm{BS}$ than node $p$, the packet is returned back to greedy mode forwarding again. In that case, the packet is forwarded to that neighbor. To do that, a node $p$ that balances a packet into recovery mode includes in the packet's header its position, i.e. the position where the packet was balanced to recovery mode. The node $p$ includes also in the header the minimum path through which the packet will be forwarded in recovery mode. Afterwards, each node $q$ that receives the packet checks first whether it has at least a neighbor closer to the BS than node $p$ using the position included in the packet's header. If so, it balances the packet to normal mode and forwards the packet to that neighbor. Otherwise, it forwards the packet according to the minimum path included in the header. Algorithm 4 summarizes routing strategy of our approach. 
Algorithm 4: Route (Packet "P")

$S i$ : the node that is handling packet $P$;

$N(\mathrm{Si})$ : the set of $S i$ 's neighbors which are closest to the BS;

$R(S i)$ : the set of Si's neighbors which are in the recovery area;

$H(S i)$ : the set of $S i$ 's one hop neighbors;

If $N(S i)==\phi$ Then $/ /$ No neighbor is closer to the $B S$;

If $S i$ is the source of the packet Then

Next_Neighbor := Nearest Neighbor in $H(S i)$; Else

If (P.mode is in normal mode) Then

Apply sub-graph $G$ ' Formation Algorithm from $R(S i)$;

Apply Prim's Algorithm;

Find the minimum path in the MST found;

Next_Neighbor $:=$ First node in the minimum path in the MST found;

Include the minimum path in the packet's header;

Else // P.mode is in recovery mode

Check the address contained in the packet's

header;

If there is at least a neighbor closer to BS than P.Rec_Addrr Then

Balance the packet to normal mode; Next_Neighbor := That_Neighbor; Else

Next_Neighbor $:=$ first node in the path included in packet's header;

\section{EndIf}

\section{EndIf}

\section{EndIf}

Else // there is neighbors closer to the BS;

Apply sub-graph $G$ ' Formation Algorithm from

$N(\mathrm{Si})$;

Apply Prim's Algorithm;

Find the minimum path in the MST found;

Next_Neighbor $:=$ First node in minimum path in the MST found;

EndIf

Transmit $P$ to Next_Neighbor;

Lemma 1: The runtime complexity of IEGGR is $(n \log m)$, where $m$ is the number of neighbors and $n$ is the number of links between these neighbors.

Proof: In IEGGR each node considers only its 1-hop neighbors which are closest to the BS to construct the local sub graph where these neighbors represent the set of vertices and the links between these neighbors represent the set of edges. With a simple implementation of Prim's algorithm, a linear search on a set of edges to find the edge with the smallest weight requires a runtime complexity of $\left(m^{2}\right)$, where $m$ is the number of vertices. However, this runtime complexity can be reduced to $(n \log n)$ by searching edges in a set of edges sorted by their weight, where $n$ is the number of edges. By searching vertices instead of edges, runtime complexity can be further improved to $(n \log m)$. Thus, the runtime complexity of IEGGR is $(n \log m)$, where $m$ is the number of neighbors and $n$ is the number of links between these neighbors.

\section{Performance Validation}

The effectiveness of IEGGR is validated through simulation. This section discusses the simulation setup, performance metrics and results.

\section{A. Simulation Environment and Performance Metrics}

To evaluate the performance of our protocol, we have used $\mathrm{J}$-Sim [58] as a simulation environment. J-Sim is a very powerful simulator in energy consumption management aspect which is the most crucial point in wireless sensor networks. Sensor nodes used in simulation are considered as homogenous : having the same initial energy quantity, the same computation capacity and memory, the same transmission range, and equipped with the same communication interface IEEE 802.11, the base station energy is considered as unlimited. These sensor nodes are deployed inside a 100 by 100 square creating topologies containing from 20 to 160 nodes. The position of generated nodes in the topologies is performed randomly. Media attenuation factor ' $\alpha$ ', linear factor ' $a$ ', and the constant ' $C$ ' used to calculate edge_weight are: $\alpha=2, a=1, C=1000$.The following table summarizes parameters used:

TABLE I

PARAMETERS USED IN THE SIMULATION

\begin{tabular}{|l|l|}
\hline \multicolumn{1}{|c|}{ Parameter } & \multicolumn{1}{c|}{ Value } \\
\hline Number of sensor nodes & $\begin{array}{l}20,40,60,80,100, \\
120,140, \text { and } 160\end{array}$ \\
\hline Topology configuration mode & Randomized \\
\hline Number of trigger nodes & 05 trigger nodes \\
\hline Network size & $100 \mathrm{~m} \times 100 \mathrm{~m}$ \\
\hline Transmission rang of sensor node & 30 meters \\
\hline Initial node energy & 0.5 Joules \\
\hline Radio Transmission power (Watt) & 0.028 watts \\
\hline Radio Reception power (Watt) & 0.036 watts \\
\hline MAC sub-layer & MAC IEEE 802.11 \\
\hline Angle $\delta$ & $2 \pi / 3$ \\
\hline
\end{tabular}

To evaluate the performances of our protocol IEGGR and its energy efficiency, we have compared it with the study we have performed before in [9]. The comparison of our protocol with these protocols will be performed with the following metrics:

- The Energy consumption: the main goal of our protocol IEGGR is to minimize node's energy consumption, because it affect directly network lifetime. That's why we will compare our protocol with other protocols in terms of average energy EM consumed by all nodes in the network:

$$
E M=\frac{\sum_{i=1}^{n}\left(\mathrm{E}_{i}-E R_{i}\right)}{\text { Total_nbr_sent_packets }}
$$

where $E_{i}$ is the initial energy of node $I, E R_{i}$ is the remaining energy of node $I$, and $n$ is the number of nodes in the network. 
- The Success rate represents the report between the number of the received packets by the base station and the total number of packets sent by all nodes in the network.

$$
\text { succes_rate }=\frac{n b r_{-} \text {packets_received_BS }}{\text { Total_nbr_sent_packets }}
$$

A routing protocol has to minimize the number of lost packets during transmission from a node to the base station, because the transported information can be very important.

- The Average latency: latency is the time required for a packet to be transmitted from the source node to the base station. Therefore, average latency is the sum of latencies of all packets divided by the total number of received packets.

$$
\text { Average_latency }=\frac{\sum_{i=1}^{n}\left(\text { reception_time }_{i}-\text { Emission_time }_{i}\right)}{n}
$$

where $n$ is the number of packets received by the base station.

\section{B. Experiment Setup and Results}

In this subsection, we present the simulation results. The individual values reflect the average of 30 independent scenarios and they are subjected to $90 \%$ confidence intervals and stay within $10 \%$ of the sample mean.

\section{1) Energy Consumption}

Fig. 6. presents the average energy consumed in relation to the number of nodes in the network whereas Fig 7 present the average energy consumed with time. From the figures we can note that IEGGR protocol improves average energy consumption in the network compared with other protocols. This improvement can be justified by the mechanism applied by IEGGR that forwards packets through the optimal path in terms of energy consumption. With EGGR protocol, the optimal path calculation is based on the assumption that the retransmission cost between each neighbor and the BS is optimal which is not the fact in the reality. This assumption degrades the performance of EGGR compared to IEGGR protocol. Other protocols (GPSR, NFP, MFR) select a neighbor closest to the BS without taking into account energy consumption criteria. This mechanism forwards packets through end to end paths that can be expensive in terms of energy consumption especially when there is a void (recovery mechanism applied by GPSR protocol to get out of voids).

Likewise, we can see from figure 6 that the number of nodes in the network increases the average energy consumed for all protocols. This can be justified by the number of control and update messages (periodic messages sent to update neighbor tables) generated in the network. On the other hand, the number of intermediate nodes in the path between the source and the base station increases average energy consumed. Indeed, the more nodes in the end-to-end path the more energy consumed. This can be noted with NFP protocol, which selects its nearest neighbor to forward packets.

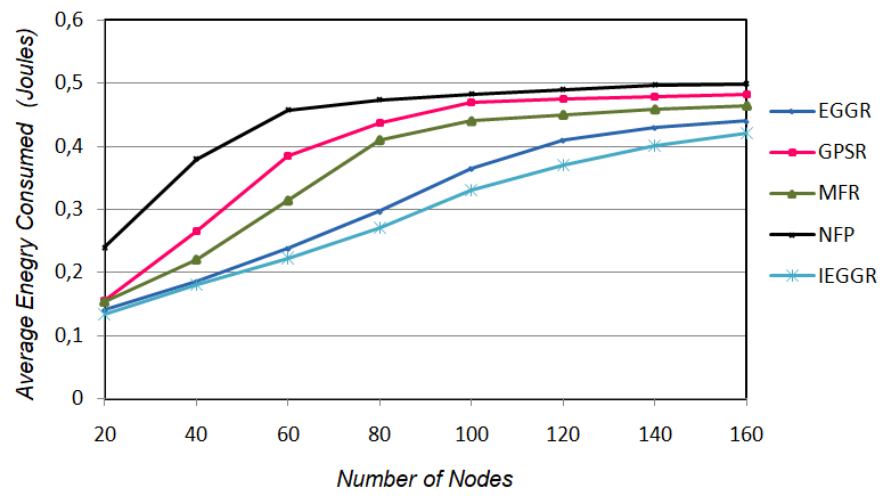

Fig. 6. Average energy consumed in relation to number of nodes in the network.

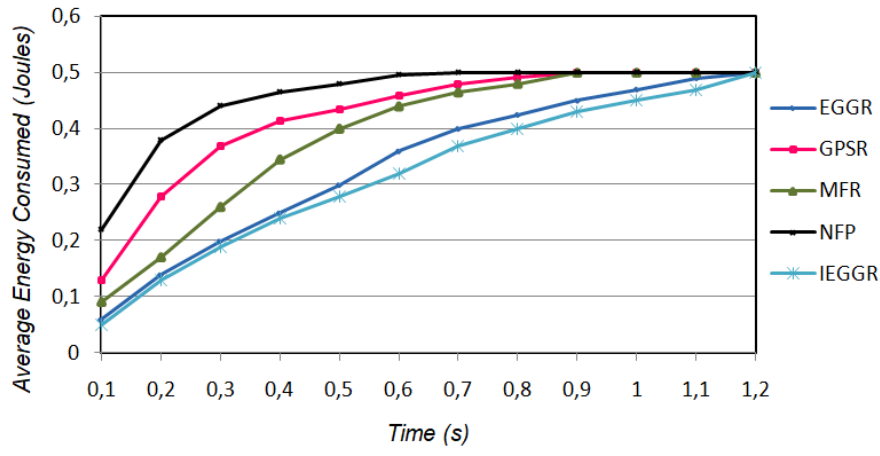

Fig. 7. Average energy consumed with time.

From figure 7, we can observe that, with other protocols, nodes consume their energy very quickly compared with EGGR protocol. And this proves the efficiency of balance mechanism applied by our protocol during shortest path computation that distributes energy consumption on all nodes efficiently which allows nodes to conserve their energy for a long time. Indeed, using a fixed criterion to select next forwarding neighbor (which is applied with NFP, MFR, and GPSR) gives the same neighbor each time. The use of the same neighbor all the time to forward packets towards the BS causes the depletion of its energy and goes down.

\section{2) Success Rate}

Figure 8 depicts the success rate marked by all protocols in relation to the number of nodes in the network. From the figure we can see that success rate of all protocols changes between $50 \%$ and $99 \%$. We can also see that the number of nodes in the network affects success rate; sure enough, the more nodes in the network the more success rate be closer to $100 \%$ for all protocols; i.e., the more nodes in the network the less probability of having voids in the network and the less nodes in the network the more probability of having voids in the network which affects success rate. We also note that the number of nodes affects success rate obtained with MFR and NFP protocols when density of the network is low which proves the necessity of using a recovery mechanism to guarantee packets delivery in WSNs. According to the simulation results, both IEGGR and EGGR give better success rates. 


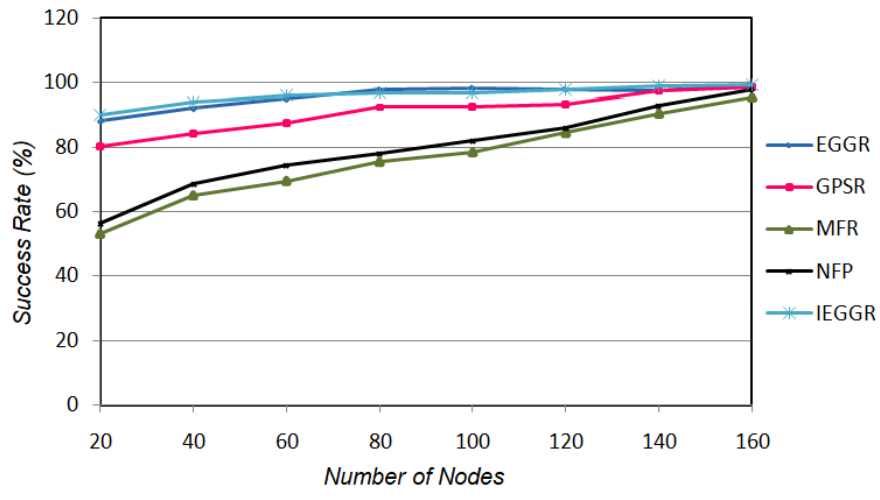

Fig. 8. Success rate.

\section{3) Network Lifetime}

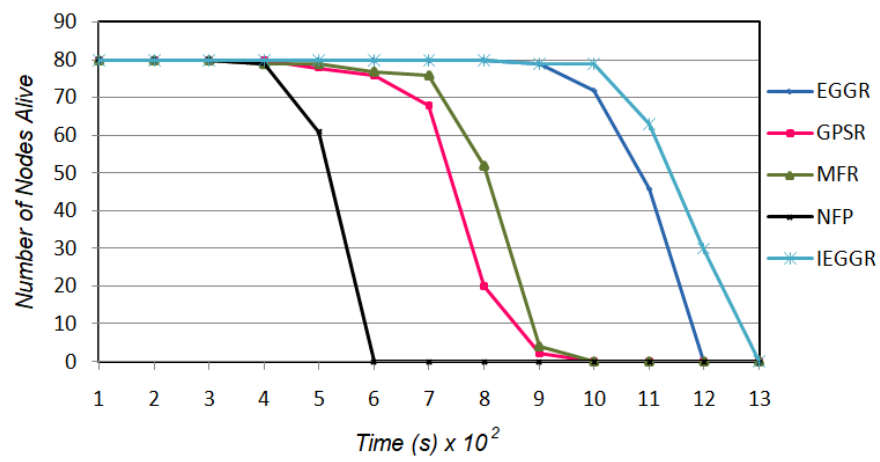

Fig. 9. Network lifetime: number of nodes alive.

Figure 9 presents the number of nodes alive with the time. From this figure, we can see that with MFR, NFP, and GPSR nodes drain their energy very quickly compared to EGGR and IEGGR protocols. This shows that without taking into account energy consumption when forwarding packets decreases the entire network lifetime. In contrast, both IEGGR and EGGR, which try to forward packets through an optimal path in terms of energy consumption, increase the entire network lifetime.

According to the results in the figure, it is clear that IEGGR allows improving traffic distribution compared to EGGR. This is because with EGGR, the optimal path calculation is based on the assumption that the retransmission cost between each neighbor and the BS is optimal. This assumption degrades the performance of EGGR compared to IEGGR protocol where the optimal path is calculated greedily as the packet progresses towards the BS.

\section{4) Average Latency}

Figure 10 shows the average end-to-end transmission delay of all protocols. From the figure, it can be noted that the end-to-end transmission delay obtained with NFP routing protocol is very high compared to the other protocols and this can be justified by the number of intermediate nodes selected by NFP protocol to forward packets from the source node to the destination. Effectively, with NFP protocol, a node selects its nearest neighbor toward the destination as next hop to forward packets. This selection strategy increases the number of hops along the path between the source and the
BS, which increases the end-to-end transmission delay. We can also see that end-to-end transmission delay obtained with MFR protocol is better than that obtained with the other protocols and this is because of recovery mechanism applied by these protocols. When the number of nodes in the network is less than or equals to 80 , the average latency obtained with IEGGR protocol is similar to that obtained with MFR, EGGR, and GPSR. However, when the number of nodes is greater than 80 , the end-to-end transmission delay obtained with IEGGR protocol is slightly greater than that obtained with these protocols. This can be justified by the number of neighbors that a node should deal with to construct the sub-graph and MST in order to find the next forwarding neighbor. According to these results, we can see that MFR protocol gives the best end-to-end transmission delay.

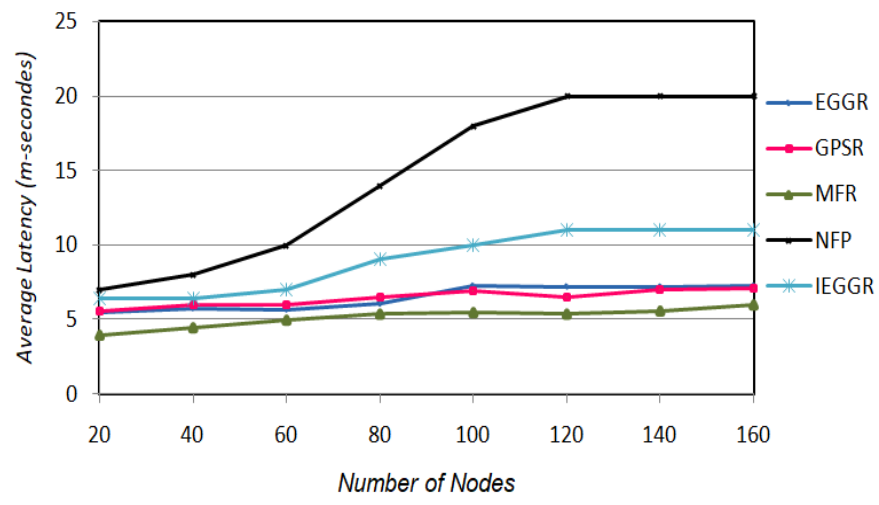

Fig. 10. End-to-end transmission delay.

\section{CONCLUSION}

The functioning of a wireless sensor networks mainly relies on multi-hop communication of nodes that cooperate with each other to connect the remote nodes with the BS. Many geographic routing protocols for wireless sensor networks have been proposed. However, some of these protocols do not take into account energy consumption. In this paper, we proposed a new geographic routing protocol that conserves nodes energy and guarantees packets delivery in wireless sensor networks based only on nodes local knowledge (neighborhood). This protocol, called IEGGR (Improved Energy-aware and delivery Guarantee Geographic Routing protocol) takes into consideration constraints imposed by sensor nodes, allowing an efficient management of energy resources during data communication in the network, by introducing a mechanism to compute the shortest path in terms of energy consumption during next node calculation. On the other hand, delivery guarantee is assured by introducing a recovery mechanism that allows forwarding packets around voids. Performances comparison between our protocol and other protocols is achieved using $\mathrm{J}$ Sim open source simulator. After achieving several simulations and analyzing obtained results, we have seen that our protocol reduces the amount of consumed energy at sensor nodes leading to entire network lifetime extension. Simulation series proved, also that our protocol (IEGGR) increases packets delivery rate between nodes and the base 
station especially in networks with high density. However, our protocol slightly influences the end-to-end transmission delay in networks with high densities. Nonetheless, the proposed approach presents a limitation, which is the problem of "very big voids". This problem happens when the entire path included in the packet's header does not allow encountering the void in recovery mode. In that case, the packet may return to the node where recovery mode has been initiated and fall down into a loop. A potential solution for such situation is to prevent returning the packet back toward that node and forwarding the packet away from that area. Another solution for such situation is to forward the packet towards the opposite direction by the node where recovery mode has been initiated. This limitation represents our future work to optimize energy consumption when forwarding packets in recovery mode to encounter very big voids.

\section{REFERENCES}

[1] H. M. A. Fahmy, "Wireless Sensor Networks," in Signals and Communication Technology, 2020, pp. 3-39. doi:10.1007/978-3-03029700-8.

[2] M.S. Obaidat, S. Misra, "Principles of wireless sensor networks," Cambridge, UK: Cambridge University Press; 2014 https://doi.org/10.1017/CBO9781139030960

[3] R.E. Mohamed, A.I. Saleh, M. Abdelrazzak, et al. "Survey on Wireless Sensor Network Applications and Energy Efficient Routing Protocols," Wireless Pers Commun 101, pp.1019-1055, 2018. https://doi.org/10.1007/s11277-018-5747-9

[4] D. Pal, S. Funilkul, N. Charoenkitkarn, and P. Kanthamanon, "Internetof-Things and Smart Homes for Elderly Healthcare: An End User Perspective," in IEEE Access, vol. 6, pp. 10483-10496, 2018, doi 10.1109/ACCESS.2018.2808472.

[5] M. Abdulkarem, K. Samsudin, F.Z. Rokhani, MF. A Rasid, "Wireless sensor network for structural health monitoring: A contemporary review of technologies, challenges, and future direction," Structural Health Monitoring 19(3), pp. 693-735, 2020. doi:10.1177/1475921719854528

[6] A. Belghith, M. S. Obaidat, "Wireless sensor networks applications to smart homes and cities," Smart Cities and Homes, pp 17-40, 2016. doi:10.1016/b978-0-12-803454-5.00002-x

[7] P. Tomar, G. Kaur, P. Singh "A Prototype of IoT-Based Real Time Smart Street Parking System for Smart Cities," In: N. Dey, A. Hassanien, C. Bhatt, A. Ashour, S. Satapathy (eds) Internet of Things and Big Data Analytics Toward Next-Generation Intelligence. Studies in Big Data, vol 30. Springer, Cham. 2018. https://doi.org/10.1007/978-3-319-60435-0_10

[8] A. Hilmani, A. Maizate, L. Hassouni, "Designing and managing a smart parking system using wireless sensor networks," in Journal of Sensor and Actuator Networks, 7(2), 24, 2018. https://doi.org/10.3390/jsan7020024

[9] M. Faheem, R. A. Butt, B. Raza, M. W. Ashraf, S. Begum, M. A. Ngadi, V. C. Gungor, "Bio-inspired routing protocol for WSN-based smart grid applications in the context of Industry 4.0," in Transactions on Emerging Telecommunications Technologies, 30(8), e3503, 2019.

[10] N. Rida, M. Ouadoud, A. Hasbi, "Traffic Signal Control for a Single Intersection-Based Intelligent Transportation System" In Digital Transformation and Innovative Services for Business and Learning (pp. 159-180). IGI Global. Singapore, 2020. doi: 10.4018/978-1-7998-51752.ch009

[11] M. A. Mondal, Z. Rehena, "An IoT-Based Congestion Control Framework for Intelligent Traffic Management System," In Advances in Artificial Intelligence and Data Engineering, pp. 1287-1297. Springer, 2021.

[12] M. Tokala, R. Nallamekala, "Secured algorithm for routing the military field data using dynamic sink: Wsn," In 2018 Second International Conference on Inventive Communication and Computational Technologies (ICICCT), pp. 471-476. IEEE, (2018, April).

[13] R. Moorthy, V. Bangera, Z. Amrin, N. J. Avinash, K. R.NS, "WSN in Defence Field: A Security Overview," In 2020 Fourth International Conference on I-SMAC (IoT in Social, Mobile, Analytics and Cloud)(ISMAC) (pp. 258-264). IEEE, (2020, October).
[14] X. Zhu, B. Ding, W. Li, et al. "On development of security monitoring system via wireless sensing network," $J$ Wireless Com Network 2018, 221, 2018. https://doi.org/10.1186/s13638-018-1235-x

[15] K. Haseeb, I. Ud Din, A. Almogren, N. Islam, "An energy efficient and secure IoT-based WSN framework: An application to smart agriculture," Sensors, 20(7), 2081, 2020.

[16] D. Kandris, C. Nakas, , D. Vomvas, G. Koulouras, "Applications of wireless sensor networks: an up-to-date survey," Applied System Innovation, 3(1), 14, 2020.

[17] J. Singh, R. Kaur, D. Singh, "A Survey and Taxonomy on Energy Management Schemes," in Wireless Sensor Networks. Journal of Systems Architecture, 101782. 2020. doi:10.1016/j.sysarc.2020.101782

[18] A. Kumar, H. Y. Shwe, K. J. Wong, P. H. Chong, "Location-based routing protocols for wireless sensor networks: A survey," in Wireless Sensor Network, 9(1), pp. 25-72, 2017.

[19] M. Singh, S. Kumar, "A survey: Ad-hoc on demand distance vector (AODV) protocol," in International Journal of Computer Applications, 161(1), pp. 38-44, 2017.

[20] J. Mulert, I. Welch, and W. K.G. Seah. "Security threats and solutions in manets: A case study using aodv and saodv". Journal of Network and Computer Applications, 35(4):1249-1259, 2012.

[21] S. A. Ade, P. A. Tijare. "performance comparison of aodv, dsdv, olsr and dsr routing protocols in mobile ad hoc networks". International Journal of Information Technology and Knowledge Management, 2(2):545-548, 2010.

[22] M. Mehic, P. Fazio, M. Voznak, "Usability of Destination-Sequenced Distance Vector Routing Protocol Routes," in 11th International Congress on Ultra Modern Telecommunications and Control Systems and Workshops (ICUMT), 2019. doi:10.1109/icumt48472.2019.8970752

[23] A. Slimani, M. Redjimi, D. Slimani, "Weighted Density Center (WDCLEACH-C) Clustering Protocol for Wireless Sensor Networks (WSN)," Informatica, 42(2), 2017.

[24] A. Slimani, M. Redjimi, D. Slimani, "An agent-based cluster head management strategy for hierarchical wireless sensor network," in International Journal of Systems, Control and Communications, 11(4), pp. $321-333,2020$.

[25] S. Tyagi, N. Kumar, "A systematic review on clustering and routing techniques based upon LEACH protocol for wireless sensor networks", in Journal of Network and Computer Applications, 36(2), pp. 623-645, 2013

[26] E. F. Ahmed, M. A. Omar, T. C. Wan, A. A. Altahir, "Work in Progress: LEACH-Based Energy Efficient Routing Algorithm for Large-Scale Wireless Sensor Networks," in Journal of Telecommunication, Electronic and Computer Engineering (JTEC), 10(1-5), pp. 83-87, 2018.

[27] B. Karp and H. Kung, "GPSR: Greedy perimeter stateless routing for wireless networks, "In Proceedings of the $6{ }^{\text {th }}$ Annual International Conference on Mobile Computing and Networking. ACM Press, pages 243-254, 2000.

[28] R.H. Milocco, H. Costantini, S. Boumerdassi. "Improved geographic routing in sensor networks subjected to localization errors". Ad Hoc Networks, 13, pp. 476-486, 2014.

[29] B. Peng, A.H. Kemp. "Energy-efficient geographic routing in the presence of localization errors". Computer Networks, 55(3), pp. 856$872,2011$.

[30] S. Lee, B. Bhattacharjee, S. Banerjee, B. Han. "A general framework for efficient geographic routing in wireless networks". Computer Networks, 54(5), pp. 844-861, 2010.

[31] M. Boulaiche, L. Bouallouche-Medjkoune. "EGGR : Energy-aware and delivery Guarantee Geographic Routing protocol". Wireless Networks, 21(6), pp. 1765-1774, 2015.

[32] S. Tao, A.L. An,anda, Mun Choon Chan, "Greedy face routing with face identification support in wireless networks", Computer Networks, 54 (2010), pp. 3431-3448, 2010.

[33] A. Kleerekoper, N.P. Filer, "Perfect link routing for energy efficient forwarding in geographic routing", Ad hoc Networks, 30, pp. 46-62, 2015.

[34] M. Al-shugran, O. Ghazali, S. Hassan, K. Nisar, A.Suki, M.Arif, "A qualitative comparison evaluation of the greedy forwarding strategies in Mobile Ad Hoc Network", Journal of Network and Computer Applications, 36, pp. 887-897, 2013.

[35] M. Z. Hasan, F. Al-Turjman, H. Al-Rizzo, "Analysis of cross-layer design of quality-of-service forward geographic wireless sensor network 
routing strategies in green internet of things," IEEE Access, 6, 2037120389,2018

[36] C. Lyu, X. Zhang, Z. Liu, C. H. Chi, „Selective authentication based geographic opportunistic routing in wireless sensor networks for Internet of Things against DoS attacks," in IEEE Access, 7, pp. 31068$31082,2019$.

[37] A. R. Hameed, S. Islam, M. ul, Raza, H. A. Khattak, "Towards energy and performance aware geographic routing for IoT enabled sensor networks" in Computers \& Electrical Engineering, 85, 106643, 2020. doi:10.1016/j.compeleceng.2020.106643

[38] S. Boussoufa-Lahlah, F. Semchedine, L. Bouallouche-Medjkoune, "Geographic routing protocols for Vehicular Ad hoc NETworks (VANETs): A survey," in Vehicular Communications, 11, pp. 20-31, 2018. https://doi.org/10.1016/j.vehcom.2018.01.006

[39] M. Al-shugran, O. Ghazali, S. Hassan, K. Nisar, A.Suki, M.Arif, "A qualitative comparison evaluation of the greedy forwarding strategies in Mobile Ad Hoc Network", i n Journal of Network and Computer Applications, 36, pp. 887-897, 2013.

[40] N. Cao, Y. Wang, J. Ding, C. Zhou, Y. Li, Y. Zhang, , ... H. Li. "The Comparisons of Different Location-Based Routing Protocols in Wireless Sensor Networks," in 2017 IEEE International Conference on Computational Science and Engineering (CSE) and IEEE International Conference on Embedded and Ubiquitous Computing (EUC). doi:10.1109/cse-euc.2017.246, 2017.

[41] A. Boukerche, B. Turgut, N. Aydin, M. Z. Ahmad, L. Boloni, D. Turgut, "Routing protocols in ad hoc networks: A survey", Computer Networks, 55, pp. 3032-3080, 2011.

[42] N. ao, P. Liu, G. Li, C. Zhang, S. Cao, G. Cao, , ... B. B. Gupta, "Evaluation models for the nearest closer routing protocol in wireless sensor networks," IEEE Access, 6, pp. 77043-77054, 2018.

[43] C. Gharat, , S. Krishnan,. "Effects of Duplicate Packet Transmission in Timer based Co-ordination Opportunistic Routing Scheme,” In 2019 International Conference on Smart Systems and Inventive Technology (ICSSIT) (pp. 401-405). IEEE, (2019, November).

[44] R. W. L. Coutinho, A. Boukerche, A. A. F. Loureiro, "A novel opportunistic power controlled routing protocol for internet of underwater things," Computer Communications. doi:10.1016/j.comcom.2019.10.020

[45] D. Chen, P. K. Varshney, "A Survey of Void Handling Techniques for Geographic Routing in Wireless Networks", IEEE Communications Surveys \& Tutorials, 9(1), pp. 50-67, 2007.

[46] M. M. Lima, H. A. B. F. Oliveira, D. L. Guidoni, A. A. F. Loureiro, "Geographic routing and hole bypass using long range sinks for wireless sensor networks," in Ad Hoc Networks, 67, pp. 1-10, 2017. doi:10.1016/j.adhoc.2017.08.010

[47] H. Huang, H.Yin, G. Min, X. Zhang, W. Zhu, Y. Wu, "Coordinateassisted routing approach to bypass routing holes in wireless sensor networks," in IEEE Communications Magazine, 55(7), pp. 180-185, 2017.

[48] K.V. Nguyen, C.H. Nguyen, P. Le Nguyen, et al. 'Energy-efficient routing in the proximity of a complicated hole in wireless sensor networks," in Wireless Netw(2021). https://doi.org/10.1007/s11276021-02569-3

[49] P. Nazareth, B.R. Chandavarkar, "Void Avoidance Node Deployment Strategy for Underwater Sensor Networks," In: Soman A., Shekhawat R., Mundra A., Srivastava S., Verma V. (eds) Smart Systems and IoT: Innovations in Computing. Smart Innovation, Systems and Technologies, vol 141. Springer, Singapore, 2020 https://doi.org/10.1007/978-981-13-8406-6_47

[50] J. A. Bondy, U. S. R. Murty. "Graph Theory with Applications". The Macmillan Press Ltd North-Holland, 1976.

[51] P. Bose, P. Morin, I. Stojmenovic, and J. Urrutia. "Routing with Guaranteed Delivery in Ad Hoc Wireless Networks". Wireless Networks, 7(6), pp. 609-616, 2001.

[52] N. K. Gupta, R. S. Yadav, R. K. Nagaria, "3D geographical routing protocols in wireless ad hoc and sensor networks: an overview," in Wireless Networks, 2019. doi:10.1007/s11276-019-01983-y

[53] J. Kuruvila, A. Nayak, and I. Stojmenovic. "Progress And Location Based Localized Power Aware Routing for Ad Hoc Sensor Wireless Networks," in International Journal of Distributed Sensor Networks, 2, pp.147-159, 2006.

[54] E. Dijkstra. "Solution of a problem in concurrent programming control". Communications of the ACM, 11(2), pp. 147$148,1968$.
[55] I. Stojmenovic and X. Lin. "Power-Aware Localized Routing in Wireless Networks," In IEEE Transactions on Parallel and Distributed Systems. 12(11), pp. 1122-1133, 2001. DOI: 10.1109/71.969123

[56] V. Rodoplu and T. Meng. "Minimum Energy Mobile Wireless Networks" in IEEE Journal on Selected Areas in Communications, 17(8), pp. 1333-1347, 1999. DOI: 10.1109/49.779917

[57] R.C. Prim, "Shortest connection networks and some generalizations", Bell System Technical Journal, 36 (6), pp. 1389-1401, 1957.

[58] http://www.physiome.org/jsim/ Accessed on 8 Dec. 2020.

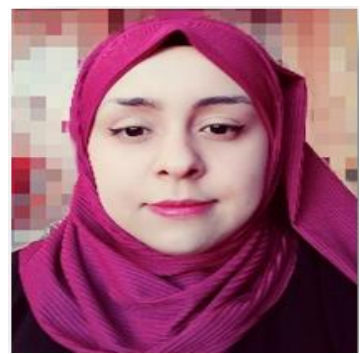

Kenza Redjimi obtained the BS. and Msc. Degrees in computer science from university 20 Aout 1955 - Skikda - Algeria respectively in 2017 and 2019. She is currently pursuing a $\mathrm{PhD}$. in computer science at the same university. Kenza Redjimi participated in several conferences and reviewed several articles. Her research interests include software engineering, modelling and simulation of industrial systems and wireless sensor networks.

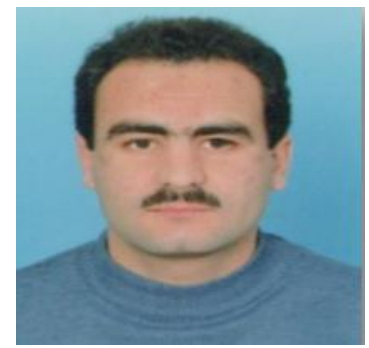

Mehdi Boulaiche received his engineer's degree in Computer Science from University of Jijel, Algeria in 2007 and Magister, $\mathrm{PhD}$ and Habilitation Universitaire (HDR) degrees in Computer Science (Networking and Distributed Systems) from University of Bejaia, Algeria in 2012, 2016 and 2020 respectively. $\mathrm{He}$ is currently an associate professor in the Department of Computer Science at the University 20 Aout 1955 , Skikda, Algeria (for courses: Distrubted systems, applications, programming and security networks). His research interests include Wireless Sensor Networks, Anonymity, Ad hoc Networks, and Security of Communication Networks. He is currently the head of the Networks and Network Security research group in Licus laboratory at universite 20 Aout 1955 - Skikda.

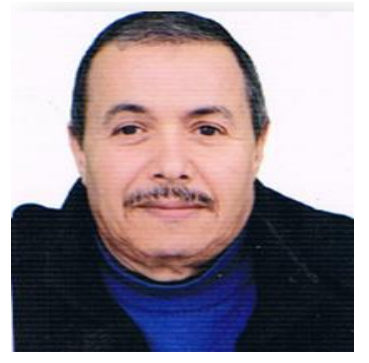

Mohammed Redjimi obtained the engineer's degree in computer science from CERI (Centre d'études et de recherches en informatique) Algiers 1981, the DEA (Diplome des études approfondies) and the doctor-Ingineer's degree in computer science from LILLE 1 university (France) respectively in 1982 and 1984 , then the Habilitation Universitaire (HDR) in computer science from Badji Mokhtar University - Annaba, Algeria in 2007.

Mohammed Redjimi was an assistant professor and researcher in computer science at Lille 1 university - Computer science department - France (19821986). He is currently a Full professor at University 20 Aout Skikda Computer science department-Algeria, and the director of the LICUS laboratory at the same university. Mohammed Redjimi publied and reviewed several scientific articles and participted in several research projects. He occupied several administrative, pedagogic and scientific functions such as dean of faculty, director of institute, head of department. His present research interests include software engineering, modelling and simulation, multi-agent systems and wireless sensor networks. 